\title{
EVALUACIÓN DEL PROYECTO DE APRENDIZAJE TUTORADO EN LA ASIGNATURA DE EDUCACIÓN PARA LA PAZ Y LA IGUALDAD
}

Project Oriented Learning assessment in Education for peace and equality subject

Avaliação do projeto de aprendizagem tutorado na disciplina de educação para a paz e igualdade

\author{
Miriam Sonlleva Velasco (1)
}

Suyapa Martínez Scott (2)

Roberto Monjas Aguado (3)

(1) Universidad de Valladolid, España. Teléfono: +34 921112294. miriam.sonlleva@uva.es

(2) Universidad de Valladolid, España. Teléfono: +34 921112294. suyapa@pdg.uva.es

(3) Universidad de Valladolid, España. Teléfono: +34 921112321. rmonjas@mpc.uva.es

\section{Resumen}

El trabajo trata de dar a conocer la experiencia de evaluación del Proyecto de Aprendizaje Tutorado en la asignatura de Educación para la Paz y la Igualdad impartida en la Facultad de Educación de Segovia en el presente curso académico. La experiencia fue realizada por 42 estudiantes del Doble Grado en Educación Infantil y Primaria, utilizando la metodología del puzle de Aronson. Los resultados muestran cómo esta forma de trabajo a través de los objetivos de la asignatura favorece las competencias autónomas de los docentes en formación, su motivación e implicación por una práctica de aula ajustada a las necesidades del alumnado y el desarrollo de una conciencia crítica que aboga por la justicia social.

Palabras clave: Educación para la paz y la igualdad; evaluación formativa; formación inicial del profesorado; proyecto de aprendizaje tutorado

\begin{abstract}
This paper tries to present the assessment experience of the Project Oriented Learning in Education for Peace and Equality subject taught in the Faculty of Education of Segovia in the current academic year. The experience was carried out by 42 Double Grade students in Early Childhood Education and Primary Education, using Aronson's puzzle methodology. The results show how this way of working through the objectives of the
\end{abstract}


subject favors the autonomous competences of the teachers in training, their motivation and implication for a classroom practice adjusted to the needs of the students and the development of a critical conscience that advocates for social justice.

Keywords: Education for peace and equality; formative assessment; initial teacher training; project oriented learning.

\section{Resumo}

Este trabalho pretende apresentar a experiência de avaliação do Projeto de Aprendizagem Tutorado na disciplina de Educação para a Paz e Igualdade lecionada na Faculdade de Educação de Segóvia no ano letivo em curso. A experiência foi realizada por 42 alunos da Licenciatura de grau duplo na Educação Pré-Escolar e Primária, utilizando a metodologia do puzzle de Aronson. Os resultados mostram como esta forma de trabalho, através dos objetivos da disciplina favorecem as competências autónomas dos docentes em formação, a sua motivação e implicação para uma prática na sala de aula ajustada às necessidades dos alunos e o desenvolvimento de uma consciência crítica que defende a justiça social.

Palavras-chave: Educação para a paz e igualdade; avaliação formativa; formação inicial de professores; projeto de aprendizagem tutorado

\section{Introducción}

El Proyecto de Aprendizaje Tutorado (PAT) es un método activo en el que los estudiantes son dirigidos por grupos para que diseñen una actuación en un contexto profesional real (Meyer, 2004). Entre sus beneficios están la promoción del aprendizaje autónomo, el desarrollo de habilidades en equipo y la creatividad (Barba, Martínez \& Torrego, 2012; Martínez-Mínguez, 2016).

\section{Contextualización}

Nuestra experiencia de evaluación se contextualiza en la asignatura de Educación para la Paz y la Igualdad de la Facultad de Educación de Segovia. Dicha asignatura se imparte en el primer curso del Programa de Estudios Conjunto de Grado en Educación Infantil y Primaria, tiene una carga de 6 créditos ECTS y se encuentra en el módulo de formación básica. Su objetivo es que el alumnado se forme en temáticas vinculadas con 
la Educación para la Paz y la Igualdad desde una mirada crítica a los acontecimientos del pasado y a la realidad actual.

El presente curso académico hemos contado con un total de 42 estudiantes matriculados. El sistema de evaluación que se desarrolla en esta materia cuenta con tres instrumentos: un portafolio o dossier de actividades, que tiene un peso en la nota final de un 35\%; un PAT, cuyo porcentaje es de un 40\% de la calificación final; y una prueba escrita de la que se deriva el $25 \%$ de la nota final de la asignatura. Los respectivos porcentajes son el resultado del consenso, realizado al comienzo del cuatrimestre, entre docentes y estudiantes sobre la calificación de la materia para el curso 2018/2019. Al considerarse que el PAT es el que representa el mayor valor, hemos decidido analizar esta experiencia como clave de la asignatura.

\section{Diseño y desarrollo}

El PAT en la asignatura Educación para la Paz y la Igualdad tiene como objetivo elaborar una intervención educativa, en forma de taller, llevarla a la práctica en un aula de Educación Infantil o Primaria y evaluar sus potencialidades y limitaciones. La propuesta se lleva a cabo entre un grupo de cinco estudiantes a través de la adaptación del puzle de Aronson (Tabla 1).

Tabla 1.

Adaptación del puzle de Aronson para el PAT de Educación para la Paz y la Igualdad

\begin{tabular}{|c|c|c|}
\hline Fases & Actuaciones de estudiantes & Actuaciones de docentes \\
\hline 1.Inicial & $\begin{array}{l}\text { Cada grupo se pondrá en contacto con el docente que } \\
\text { tutela al alumnado con el que se realizará el taller y se } \\
\text { entrevistará con él. El objetivo de esta entrevista es } \\
\text { conocer qué se está trabajando en el aula, tener una } \\
\text { referencia del alumnado y el contexto y consensuar la } \\
\text { temática del proyecto vinculada con la asignatura. }\end{array}$ & $\begin{array}{l}\text { Buscar el centro en el que se } \\
\text { pondrán en práctica los } \\
\text { talleres, contactar con el } \\
\text { docente de referencia, orientar } \\
\text { la entrevista y supervisar su } \\
\text { correcto desarrollo. }\end{array}$ \\
\hline $\begin{array}{l}\text { 2.Formación de } \\
\text { expertas }\end{array}$ & $\begin{array}{l}\text { Cada persona del grupo asume el rol de experta en un } \\
\text { tema (valores, cuentos, igualdad, juegos y recursos } \\
\text { web). Después elabora un informe y comparte con el } \\
\text { grupo la información. }\end{array}$ & $\begin{array}{l}\text { Facilitar lecturas para } \\
\text { expertas. Orientar, tutorizar y } \\
\text { evaluar el informe. }\end{array}$ \\
\hline $\begin{array}{l}\text { 3.Programación } \\
\text { del taller }\end{array}$ & $\begin{array}{l}\text { La información de las expertas es la base para la } \\
\text { programación de un taller que debe estar relacionado } \\
\text { con un bloque de la asignatura e incluir: justificación, } \\
\text { objetivos y contenidos, marco teórico, relación con el } \\
\text { currículum, metodología, propuesta de actividades, } \\
\text { evaluación y continuidad en el tiempo. }\end{array}$ & $\begin{array}{l}\text { Dar directrices básicas para la } \\
\text { programación, revisar su } \\
\text { correcta elaboración y } \\
\text { orientar a los estudiantes para } \\
\text { que el documento escrito } \\
\text { responda a los criterios de } \\
\text { evaluación de la rúbrica. }\end{array}$ \\
\hline $\begin{array}{l}\text { 4.Puesta en } \\
\text { práctica }\end{array}$ & $\begin{array}{l}\text { Realización del taller en un aula de Infantil o Primaria } \\
\text { y observación de otra propuesta de un grupo de } \\
\text { compañeros. }\end{array}$ & $\begin{array}{l}\text { Coordinación con docentes } \\
\text { tutores de la escuela, } \\
\text { supervisión de los talleres y } \\
\text { apoyo de la puesta en } \\
\text { práctica. }\end{array}$ \\
\hline
\end{tabular}

Evaluación del proyecto de aprendizaje tutorado en la asignatura de Educación para la Paz y la Igualdad 


\begin{tabular}{|c|c|c|}
\hline $\begin{array}{l}\text { 5.Elaboración } \\
\text { definitiva }\end{array}$ & $\begin{array}{l}\text { Tras la realización del taller se pueden realizar } \\
\text { cambios en la programación, en función de la } \\
\text { experiencia vivida y se debe añadir la evaluación de } \\
\text { este. }\end{array}$ & $\begin{array}{llr}\text { Orientar y guiar las } \\
\text { propuestas finales para } \\
\text { correcta entrega. }\end{array}$ \\
\hline 6.Evaluación & $\begin{array}{l}\text { Consiste en la defensa del proyecto en el aula, que } \\
\text { será evaluada por los compañeros de otros grupos y } \\
\text { los docentes. Además, cada miembro del grupo } \\
\text { deberá entregar una autoevaluación de su } \\
\text { participación en el PAT. }\end{array}$ & $\begin{array}{l}\text { Dinamizar la sesión de } \\
\text { evaluación, dar feedback a } \\
\text { todas las exposiciones y } \\
\text { elaborar el informe de } \\
\text { evaluación. }\end{array}$ \\
\hline
\end{tabular}

Fuente: elaboración propia

El PAT tiene un porcentaje del $40 \%$ de la calificación de la asignatura, dentro del cual otorgamos un $10 \%$ al informe de expertas; un $20 \%$ a la programación del taller y un $10 \%$ a la puesta en práctica y a la defensa del proyecto en el aula. Además, para poder evaluar la propuesta es necesaria la entrega de la entrevista con el docente y el cuestionario de autoevaluación (ANEXO I).

\section{Evaluación}

En cuanto a la evaluación de la experiencia, los 42 estudiantes aprobaron esta parte de la asignatura con calificaciones superiores a 6,5 puntos. Consideramos que el trabajo en grupo y la asunción de roles dentro del mismo favoreció que el alumnado sacara lo mejor de sí mismo. Si bien en el informe de expertas no todos los alumnos pusieron el mismo interés en su correcta elaboración, sí lo hicieron con la programación del taller, la puesta en práctica y la defensa. Además, más del $80 \%$ de los cuestionarios del alumnado dan muestras de que el PAT les sirvió para aprender a programar de forma contextualizada (resultados de las preguntas 3 y 6 del cuestionario); para ser críticos con sus propias actuaciones como docentes (resultados de las preguntas 2, 4, 6 y 7 del cuestionario); y para valorar la importancia de una educación capaz de adaptarse a las necesidades del alumnado y de propiciar la justicia social, objetivos propios de la asignatura (resultados de las preguntas 1 y 8 del cuestionario).

\section{Conclusiones}

Como conclusiones de la experiencia destacamos la importancia de: a) generar experiencias que permitan al profesorado en formación tener un contacto real con la escuela y lo que ocurre dentro de ella; b) realizar propuestas en equipos de trabajo, como forma de fomentar el aprendizaje cooperativo (Slavin, 1999); y c) llevar a cabo en el aula programaciones asentadas en temáticas como los Derechos Humanos, el Género, 
la Comprensión Internacional, la Interculturalidad o la Educación para el Desarrollo, que favorecen una educación justa y democrática.

\section{Referencias}

Barba, J.J., Martínez, S., \& Torrego, L. (2012). El proyecto de aprendizaje tutorado cooperativo. Una experiencia en el grado de maestra de Educación Infantil. Revista de Docencia Universitaria, 10(1), 123-144.

Martínez-Mínguez, L. (2016). Proyectos de Aprendizaje Tutorados y autoevaluación de competencias profesionales en la formación inicial del profesorado. Retos, 29, 242-250.

Meyer, V. (2004). Project oriented learning (POL) as a communication tool of environmental sciences in the community of Sohanguve. A case study. Recuperado de: www.saasta.ac.za/scicom/pcst7/meyer_v.pdf

Slavin, R.E. (1999). Aprendizaje cooperativo. Teoría, investigación y práctica. Argentina: Aique. 


\section{ANEXO I. Cuestionario de autoevaluación del PAT en la asignatura Educación para la Paz y la Igualdad}

\section{TÍTULO DEL PROYECTO:}

Nombre y apellidos:

Nombres de tus compañeras o compañeros:

Valora del 1 al 5 (1= mínimo; 5= máximo) los siguientes aspectos del trabajo en grupo (G es la valoración del grupo en general; $\mathrm{P}$ se refiere a tu propia valoración como miembro del grupo):

1) El aprendizaje que he o hemos obtenido. Puntuación: Mis razones para la valoración:

\begin{tabular}{|l|l|l|l|l|l|}
\hline $\mathbf{G}$ & $\mathbf{1}$ & $\mathbf{2}$ & $\mathbf{3}$ & $\mathbf{4}$ & $\mathbf{5}$ \\
\hline $\mathbf{P}$ & $\mathbf{1}$ & $\mathbf{2}$ & $\mathbf{3}$ & $\mathbf{4}$ & $\mathbf{5}$ \\
\hline
\end{tabular}

2) La organización en el grupo para realizar el trabajo y mi aportación a la misma.

\begin{tabular}{|l|l|l|l|l|l|}
\hline $\mathbf{G}$ & $\mathbf{1}$ & $\mathbf{2}$ & $\mathbf{3}$ & $\mathbf{4}$ & $\mathbf{5}$ \\
\hline $\mathbf{P}$ & $\mathbf{1}$ & $\mathbf{2}$ & $\mathbf{3}$ & $\mathbf{4}$ & $\mathbf{5}$ \\
\hline
\end{tabular}

Mis razones para la valoración:

3) La presentación final del trabajo por escrito Mis razones para la valoración

\begin{tabular}{|l|l|l|l|l|l|}
\hline $\mathbf{G}$ & $\mathbf{1}$ & $\mathbf{2}$ & $\mathbf{3}$ & $\mathbf{4}$ & $\mathbf{5}$ \\
\hline $\mathbf{P}$ & $\mathbf{1}$ & $\mathbf{2}$ & $\mathbf{3}$ & $\mathbf{4}$ & $\mathbf{5}$ \\
\hline
\end{tabular}

4) El desarrollo de los distintos contenidos del proyecto Mis razones para la valoración:

\begin{tabular}{|l|l|l|l|l|l|}
\hline $\mathbf{G}$ & $\mathbf{1}$ & $\mathbf{2}$ & $\mathbf{3}$ & $\mathbf{4}$ & $\mathbf{5}$ \\
\hline $\mathbf{P}$ & $\mathbf{1}$ & $\mathbf{2}$ & $\mathbf{3}$ & $\mathbf{4}$ & $\mathbf{5}$ \\
\hline
\end{tabular}

5) ¿Cuántas horas has dedicado tú a este trabajo de grupo?

5.1) ¿Cuántas reuniones se han realizado?

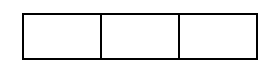

5.2) ¿A cuántas has asistido?

6) Mi aportación al trabajo ha sido (explica en qué aspectos concretos del trabajo has intervenido y qué tareas has realizado):

7) Califícate del 0 al 2 siendo cero la mínima calificación y 2 la máxima calificación en relación a la puesta en práctica del PAT en el colegio.

8) Explica un aspecto que deba permanecer de esta forma de trabajar y otro que deba ser cambiado.

9) Completa la tabla:

Evaluación del proyecto de aprendizaje tutorado en la asignatura de Educación para la Paz y la Igualdad 


\begin{tabular}{|c|c|c|c|c|c|}
\hline Afirmaciones a valorar & $\begin{array}{l}\text { Nada de } \\
\text { acuerdo }\end{array}$ & $\begin{array}{l}\text { Poco de } \\
\text { acuerdo }\end{array}$ & $\begin{array}{c}\text { Bastante de } \\
\text { acuerdo }\end{array}$ & $\begin{array}{l}\text { Totalmente } \\
\text { de acuerdo }\end{array}$ & Observaciones \\
\hline $\begin{array}{l}\text { El trabajo mediante el } \\
\text { Puzle de Aronson mejora } \\
\text { las limitaciones de los } \\
\text { trabajos grupales con } \\
\text { respecto a la evaluación } \\
\text { individual }\end{array}$ & & & & & \\
\hline $\begin{array}{l}\text { Es importante el papel del } \\
\text { docente en los seminarios }\end{array}$ & & & & & \\
\hline $\begin{array}{l}\text { Es importante el trabajo en } \\
\text { los seminarios }\end{array}$ & & & & & \\
\hline $\begin{array}{l}\text { El grupo de expertas ha } \\
\text { servido para compartir } \\
\text { información }\end{array}$ & & & & & \\
\hline $\begin{array}{l}\text { El aprendizaje conseguido } \\
\text { en el grupo } \\
\text { de expertas ha sido muy } \\
\text { alto }\end{array}$ & & & & & \\
\hline $\begin{array}{l}\text { Sola habría conseguido } \\
\text { ese nivel de aprendizaje }\end{array}$ & & & & & \\
\hline $\begin{array}{l}\text { Mi aportación individual } \\
\text { al trabajo en grupo ha sido } \\
\text { importante }\end{array}$ & & & & & \\
\hline $\begin{array}{l}\text { El grupo de trabajo ha } \\
\text { servido para compartir } \\
\text { información }\end{array}$ & & & & & \\
\hline $\begin{array}{l}\text { El aprendizaje conseguido } \\
\text { en el grupo de trabajo ha } \\
\text { sido muy alto }\end{array}$ & & & & & \\
\hline $\begin{array}{l}\text { La puesta en práctica del } \\
\text { taller en un aula de } \\
\text { Educación Infantil es una } \\
\text { parte decisiva del proyecto }\end{array}$ & & & & & \\
\hline
\end{tabular}

